\title{
Staging Models in Bipolar Disorder: A Systematic Review of the Literature
}

\author{
Ather Muneer \\ Department of Psychiatry, Islamic International Medical College, Riphah International University, Rawalpindi, Pakistan
}

\begin{abstract}
Bipolar disorder is manifested as severe dysregulation of mood with recurrent manic and major depressive episodes. It is associated with psychiatric and medical comorbidities, inadequate response to currently available pharmacological agents and a progressively deteriorating course in many patients. The index episode is often depressive in nature, while the first manic or hypomanic episode may occur several years later in the course of the disorder causing delay in diagnosis and use of inappropriate treatment strategies. Staging has been used to great advantage in other branches of medicine like cardiology and oncology. There is growing realization that major mental disorders are fundamentally progressive, with simpler treatment requirements and better prognosis during initial stages of the illness. Defining these conditions into clinically applicable stages not only helps in better understanding the trajectory of a particular disorder, but also assists in management. Patients with a chronic, recalcitrant condition like bipolar disorder are likely to greatly benefit from this approach. If the illness is correctly identified early in its course, proper treatment can be instigated arresting progression to latter phases which are associated with myriad complications in the biopsychosocial realm. With these considerations, a search of the MEDLINE data base was conducted to seek out literature pertaining to staging models in bipolar disorder. A thorough scrutiny of the existing research work revealed that a number of investigators have endeavored to stage define bipolar disorder. This paper outlines staging proposals for bipolar disorder which have the greatest supporting evidence in the literature.
\end{abstract}

KEY WORDS: Bipolar disorder; Staging; Neuroprogression; Allostatic load; Biomarkers.

\section{INTRODUCTION}

Bipolar disorder (BD) is a serious neuropsychiatric condition characterized by chronic instability of mood, circadian rhythm disturbances and continual fluctuations in energy levels, affect, sleep and cognitive appraisal of the self and others. ${ }^{1)}$ Many patients suffer their first mood episode in adolescence or early adulthood and the disease follows a persistent course spanning the entire life, well beyond age 70 years. ${ }^{2)}$ Bipolar diathesis has a pervasive adverse effect on all aspects of patients' lives as they experience social, psychological, academic and occupational difficulties due to the underlying disease. Since BD is a lifelong disorder, there is an immense unmet need to stage define the illness which helps in better understanding the disease trajectory as well as delivering personalized treatments to the patients.

\footnotetext{
Received: July 13, 2015 / Revised: August 31, 2015

Accepted: September 1, 2015

Address for correspondence: Ather Muneer, MD

Department of Psychiatry, Islamic International Medical College, Riphah International University, 274 Peshawar Road, Rawalpindi, Pakistan

Tel: +92-51-5481828, Fax: +92-51-5125170

E-mail: muneerather2@gmail.com
}

$\mathrm{BD}$ is a progressive condition which has a prodrome, a symptomatic phase and a residual state. ${ }^{3)}$ Many authors have underscored the problem of delay in initial identification which is of the magnitude of several years in some cases due to misdiagnosis and giving of wrong labels to patients with the bipolar phenotype. ${ }^{4)}$ Inappropriate or inadequate treatment can result in repeated mood episodes, persistent subthreshold symptoms, development of comorbidities such as substance use disorders, and progression of the disease with cognitive impairment and functional decline. ${ }^{5)}$ There is mounting evidence that manic and major depressive episodes (MDE) have a toxic effect on neurons and glia, causing permanent damage to areas of the brain critical in mood regulation. ${ }^{6)}$ The neuroprogressive nature of $\mathrm{BD}$ can be arrested with early diagnosis, proper treatment on the biopsychosocial lines, recognition of neuropsychiatric and physical comorbidities and individually tailored management according to the phase of the illness. ${ }^{7)}$ In this respect it is imperative to demarcate the stages of the disorder in order to facilitate the delivery of improved patient care and attain better outcomes for the sufferers of this recalcitrant condition. ${ }^{8)}$ It must be kept in mind that the staging approach has been

() This is an Open-Access article distributed under the terms of the Creative Commons Attribution Non-Commercial License (http://creativecommons.org/licenses/by-nc/4.0) which permits unrestricted non-commercial use, distribution, and reproduction in any medium, provided the original work is properly cited. 
highly beneficial in the overall management of other complex (gene $\mathrm{x}$ environment) disorders including diabetes, cardiovascular disease and various forms of cancer.

Staging presupposes that illness advances in an identifiable temporal progression of phases from an at-risk, prodromal period to the first clinical manifestations, and further developing into more severe, intractable forms with evolution and chronicity. ${ }^{9)}$ Current diagnostic systems emphasize presenting symptoms with a categorical and cross-sectional approach without considering the context, the preceding risk factors and neglecting what is known about the natural history of illness development. ${ }^{10)}$ More than a century ago, Kraepelin first realized that psychiatric disorders had a staged development and his view of their progressive nature was reflected in his nomenclature of dementia praecox and manic depressive insanity. Regarding the latter, he recognized that while the initial episodes were frequently precipitated by stressful events followed by complete improvement, as the illness evolved the patients had affective recurrences in the absence of stressful experiences and did not show full inter-episode recovery. ${ }^{11)}$

Clinical staging is a useful conceptual framework to refine the phenotype of illness along developmental lines and supports the identification of trait and state specific biomarkers. It paves the way for stratified and personalized treatment at each stage of the illness as is happening in other branches of medicine. ${ }^{12)}$ With regards to a progressive condition like BD early intervention is likely to be simpler and more effective and aims to significantly prevent or delay advancement to more severe presentations requiring complex treatment strategies which are less acceptable to patients and have a lower benefit-to-risk ratio. ${ }^{13)}$ In spite of the obvious benefits of clinical staging, this aspect of illness classification has been neglected in psychiatry until recently. However, there is now an increased impetus to characterize by stages major psychiatric disorders, including BD. In this respect, staging models have been proposed by different researchers incorporating the longitudinal history of BD from a high, at-risk period to illness progression to more severe and refractory states. The purpose of this manuscript is to systematically review the extant literature regarding the staging schemes put forward by key investigators in the field and provide an overview of this innovative and exciting area of research.

\section{MAIN SUBJECTS}

\section{Search Strategy}

The MEDLINE data base was searched with the terms "Bipolar disorder and staging", "Bipolar disorder and progression" and "Bipolar disorder and neuroprogression". A total of 746 items were retrieved dating from January 1970 to June 2015. Of these, 40 records were excluded because of the duplicate nature. Among the remaining 706 citations, a further 70 items were eliminated because these were not in English language. The titles of the left over 636 records were examined for their relevance to the staging of BD. This approach resulted in the ruling out of further 420 records from the study; the rationale was to exclude case studies, investigations carried out on animals, papers describing specific pharmacological or psychological interventions, and items that had the major focus on other psychiatric disorders. The abstracts of the remaining two hundred and sixteen articles were read and the significance of the studies with regards to parameters of treatment, functionality and biomarkers for BD was judged. Twenty eight studies qualified for final inclusion that comprised of general reviews, systematic reviews, meta-analyses, prospective and cross-sectional studies. These were read in full and the reference lists of the said articles were also consulted for additional information that could have impact on proposed staging models for BD. See Fig. 1 for a summary of this investigative appraisal of the extant literature.

\section{Developmental Trajectory and Clinical Heterogeneity of Bipolar Disorder}

\section{Classical, recurrent bipolar disorder}

$\mathrm{BD}$ is currently viewed as a spectrum illness which includes several phenotypes. The classic BD is typified by recurrent mood episodes of opposite polarity with full inter-episode recovery, very high heritability, and a good response to lithium in up to $80 \%$ of the cases. ${ }^{14)}$ It is not associated with cognitive and functional decline and is characterized by lack of psychiatric comorbidities like anxiety and substance use disorders. The family history of patients with classical BD is marked by high genetic predisposition in that they usually have a parent or a close relative with a similar condition. ${ }^{15)}$ As children and adolescents they may show an excess of adjustment, sleep and anxiety disorders such as circadian rhythm sleep disorders and separation anxiety disorder. The offspring of classical, lithium-responsive bipolar patients are often gifted, 


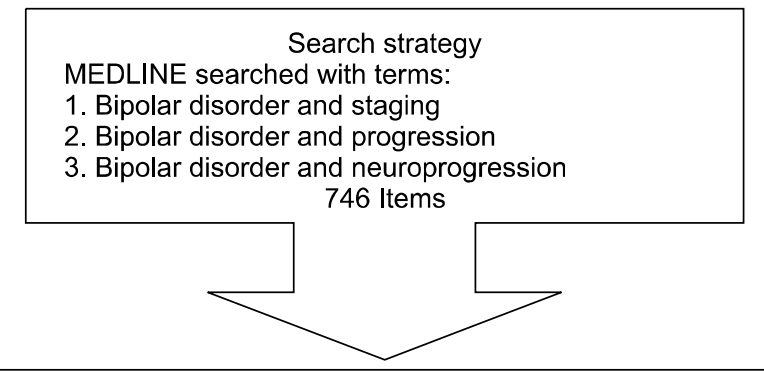

40 Citations excluded because of duplicate nature.

Further 70 items left out because those were not in English ianguage.

The titles of the remaining 636 articles were examined for their relevance to the staging of bipolar disorder. This resulted in the ruling out of further 420 records.

Excluded items:

1) Case reports

2) Animal investigations

3) Papers describing specific pharmacological/ psychological interventions

4) Articles with major focus on other psychiatric disorder
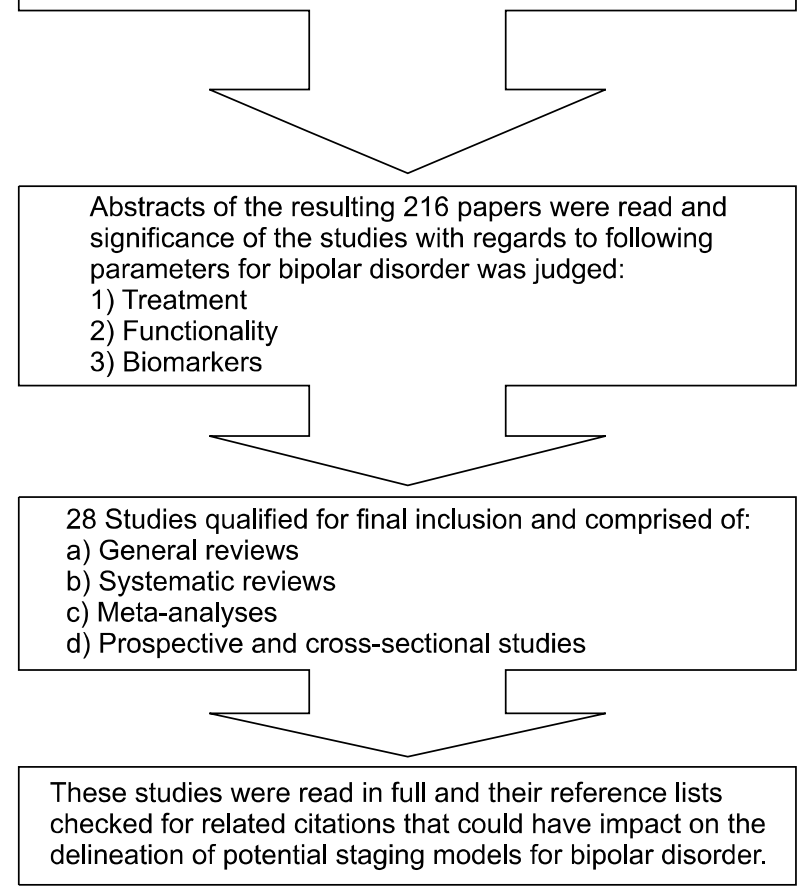

Fig. 1. Flow diagram of the search strategy used in the preparation of the systematic review.

have a completely normal early development with good academic and social adjustment. ${ }^{16)}$ In rare cases classic, lithium-responsive BD may occur in individuals without a family history of the condition but this would be an exception rather than the rule. The frequency of recurrences and the quality of remission are known to vary substan- tially between patients; however, over time there is no significant change in illness phase and polarity, absence of rapid cycling and lack of sub-threshold affective symptoms between episodes. ${ }^{17)}$ This phenotype is marked by an absence of evidence for progressive worsening of the course, and a high incidence of recurrent mood disorders but not schizophrenia in the relatives. ${ }^{18)}$ In one sample of outpatients, this comprised of $30 \%$ of the total bipolar patients seen in a subspecialty clinic of a Canadian teaching hospital. ${ }^{19)}$ The classical episodic lithium responsive subtype now forms part of a much broader spectrum of BD.

\section{Bipolar spectrum disorders}

Evidence supports that bipolar subtypes defined by either prophylactic response or nonresponse to lithium differ in clinical course, family history and neurobiological correlates. ${ }^{20)}$ Lithium nonresponsive subtypes are more chronic disorders that preferentially respond to atypical neuroleptics (psychotic spectrum) or to anticonvulsants (BD type II, comorbid anxiety disorders). ${ }^{21)}$ Psychotic spectrum BD has overlapping neurobiological findings with psychotic disorders and a higher risk of chronic nonepisodic illnesses in family members, including schizophrenia. ${ }^{22)}$ In help seeking symptomatic youth, the illness trajectory of non-classical BD is marked by negative symptoms like affective flattening, anhedonia, apathy and social withdrawal, overlapping clinically with depressive spectrum presentations. ${ }^{23)}$ They are liable to neurodevelopmental disorders including learning disabilities, attentional problems, academic failure and may exhibit cluster A personality traits. ${ }^{24)}$ The illness course of young bipolar patients may be progressive, marked by mixed episodes, incomplete remissions, persistent subthreshold symptoms, cognitive impairments and functional decline in the latter stages of the illness. ${ }^{25)}$ There is evidence for a continual pro-inflammatory state, increased level of oxidative stress and failing neurotrophic support. ${ }^{26)}$ Neuroimaging studies show increased volume of lateral ventricles with loss of brain matter in the final stages of the disease.

\section{Youth precursors of bipolar disorder}

$\mathrm{BD}$ is not diagnosed until the occurrence of the first activated manic/hypomanic episode; however, the bipolar diathesis most often starts with a depressive episode which may antecede the first diagnosable activated episode by many years. A positive family history informs the risk of BD in youth who present with melancholic depressive episodes. ${ }^{27)}$ There are other antecedents which in- 
clude non-specific anxiety syndromes, and persistent mood instability diagnosable only as depressive disorder not otherwise specified, cyclothymia, or emotional dysregulation. ${ }^{28)}$ In addition, in samples of help seeking youth attenuated psychotic symptoms, attentional and cognitive problems often co-exist with affective symptoms. ${ }^{29)}$ These early forerunners are nonspecific and are seen in other illness trajectories including psychotic disorders, internalizing and externalizing disorders, and substance use disorders. ${ }^{30)}$ These can also be self-limited presentations in some young people and do not progress to major psychiatric illnesses. However, it is the nature of the family history that brings important context to the otherwise vague clinical manifestation in children and adolescents. The history of $\mathrm{BD}$ in close family members and their response to specific treatments, for example lithium are important predictive factors aiding in the early differentiation of illness trajectories and in foretelling treatment outcome in symptomatic youth. For instance, stimulants and antidepressants should be either avoided or, if used kept to low doses, short duration and closely monitored in patients with a confirmed family history of $\mathrm{BD} .^{31)}$

\section{Prospective studies of offspring of parents with bipolar disorder}

A number of longitudinal, prospective studies of offspring of parents with confirmed diagnosis of BD have shown divergence in illness course based on subtypes of the illness. Specifically, children of parents with BD with an excellent response to long-term lithium had a completely normal or endowed early developmental course. In contrast, the children of parents who failed to respond to lithium prophylaxis manifested childhood problems with cognition, emotional regulation, socialization, and showed neurodevelopmental disorders. ${ }^{32)}$ Among high-risk offspring who developed major mood disorders, the children of lithium responders had an episodic, remitting course and responded to lithium prophylaxis, while the offspring of lithium nonresponders exhibited a non-fully remitting course and responded preferentially to either prophylaxis with an anticonvulsant or an atypical antipsychotic. ${ }^{33)}$ Moreover, the spectrum of end-stage disorders among the affected offspring of lithium responders was limited to mood disorders, while the offspring of lithium nonresponders showed both mood and psychotic spectrum illnesses. ${ }^{34)}$ The analysis, based on up to 17 years of prospective clinical research observation, holds up that BD develops in a sequence of predictable clinical phases; not all offspring manifest all stages, but once they enter the model they proceed in a forward sequence. The clinical staging model is a collective overview, and it should be kept in mind that not all high-risk offspring follow the model or advance to end-stage illness.

\section{Stage 0}

Well but at confirmed familial risk for classical BD
Stage 1

Episodic anxiety/sleep disorders
Stage 2

Single depressive episode depression-NOS, dysthymia, cyclothymia, adjustment disorders

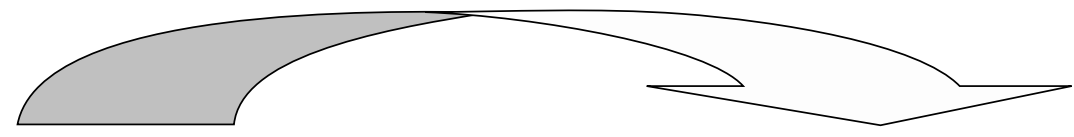

Stage 3

Recurrent melancholic MDD with or without psychotic features

Only with a family history of bipolar disorder

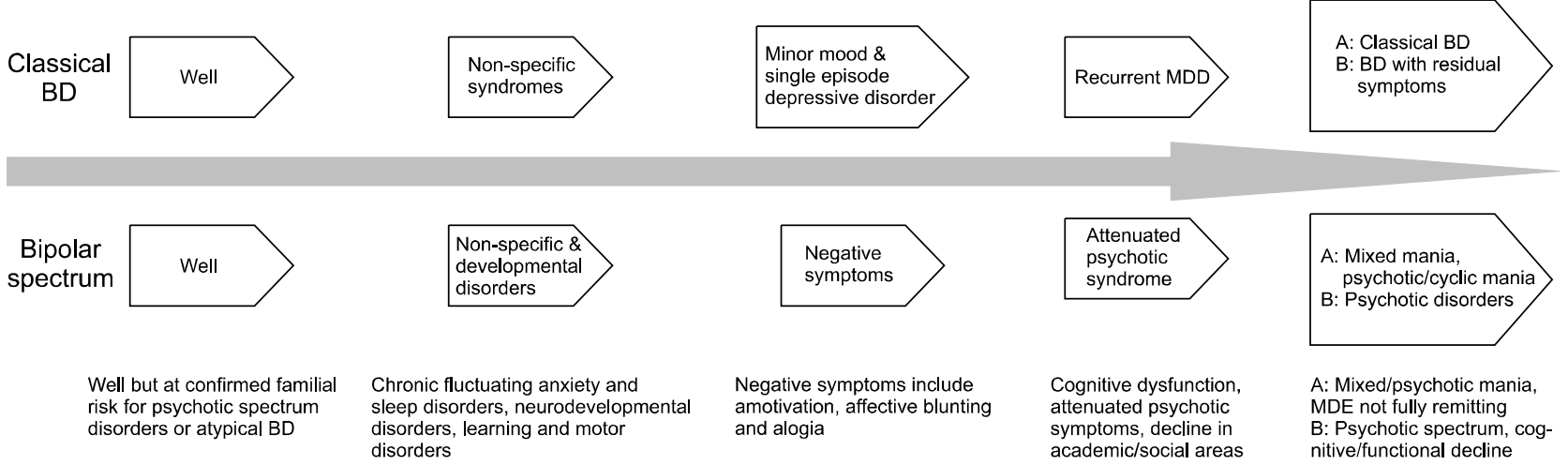

Fig. 2. Duffy's integrative clinical staging model for bipolar disorder.

BD, bipolar disorder; MDD, major depressive disorder; MDE, major depressive episode; NOS, not otherwise specified. 


\section{Staging Model Proposed by Duffy ${ }^{35)}$}

Based on above observations, Duffy has come forward with a staging scheme that emphasizes the family history, early childhood precursors, and adolescent psychopathology. This model defines the course and prognosis of $\mathrm{BD}$ according to illness subtypes and is helpful in predicting the latter stages of the diathesis in subsets of patients. ${ }^{35)}$ A schematic representation of her version is provided in Fig. 2.

\section{Neuroprogression as the Basis for Staging in Bipolar Disorder}

There is ample research evidence that $\mathrm{BD}$ is an evolving condition in which early stages have different clinical features compared to latter phases. The advancement of the illness, frequently connected with inadequate treatment is associated with a higher number of episodes occurring spontaneously as the disease progresses. ${ }^{36)}$ There is a greater rate of medical and psychiatric comorbidities, increased risk of suicide, more number of hospitalizations and poorer response to treatment. ${ }^{37)}$ Accompanying these phenomena are cognitive deterioration and functional decline in such areas as occupation, social relationships, and financial independence with eventual loss of autonomy. ${ }^{38)}$ Research efforts in the last few decades have started to uncover the neurobiological correlates of the worsening clinical course in BD. The term "neuroprogression" has been increasingly used to define the pathological reorganization of the central nervous system (CNS) which arises as the result of several insults, such as inflammation and oxidative stress. ${ }^{39)}$ In BD, neural substrate reactivity is changed by repeated mood episodes ultimately promoting a brain rewiring that leads to an increased vulnerability to life stresses. An episode-dependant deterioration pattern has been widely described in serum biomarkers, brain imaging and neuropsychological performance in effected individuals. ${ }^{40)}$ Staging which attempts to delineate the illness trajectory into phases can be considered as another facet of neuroprogression in BD. This conceptual framework is illustrated in Fig. 3.

\section{Allostatic Load as the Cause of Progression in Bipolar Disorder}

$\mathrm{BD}$ is a complex and multifactorial disease with genetic and environmental factors contributing to its clinical expression. BD can also be regarded as an illness involving the cumulative build-up of allostatic states, where physiological 'wear and tear' described as "allostatic load (AL)" progressively increases as stressors and mood epi- sodes occur over time. ${ }^{41)}$ Affective episodes acting as allostatic states generate a burden that is responsible for illness progression commonly seen in $\mathrm{BD} .{ }^{42)} \mathrm{AL}$ may contribute to a better understanding of the diathesis, particularly to the inter-episode phenomena such as susceptibility to drug and alcohol abuse, decline in psychosocial functioning, cognitive dysfunction and affliction with cardiovascular, metabolic and endocrine diseases. Bipolar patients exhibit alterations in major mediators of AL, for instance persistent dysregulation of the hypothalamicpituitary-adrenal (HPA) axis, modified immune response with a continual pro-inflammatory state, increased damage due to the occurrence of oxidative stress and decreased neuronal survival as a result of withdrawal of neurotrophic support. ${ }^{43)}$ Greater during the acute phases of the disease, these deleterious changes remain sub-threshold even during remission and damage the CNS, as the elements of brain tissue are particularly vulnerable to the mediators of AL. Whereas, the body attempts to maintain homeostatic balance in the presence of ever mounting stress, increasing AL overwhelms the compensatory mechanisms causing permanent damage to the brain and the peripheral organs. ${ }^{44)}$ Fig. 4 depicts how intermediary factors of AL act in an orchestrated harmful manner inflicting incremental damage to the whole body.

\section{Biomarkers of Bipolar Disorder}

Potential neuroimaging markers

Many studies have reported the existence of changes in

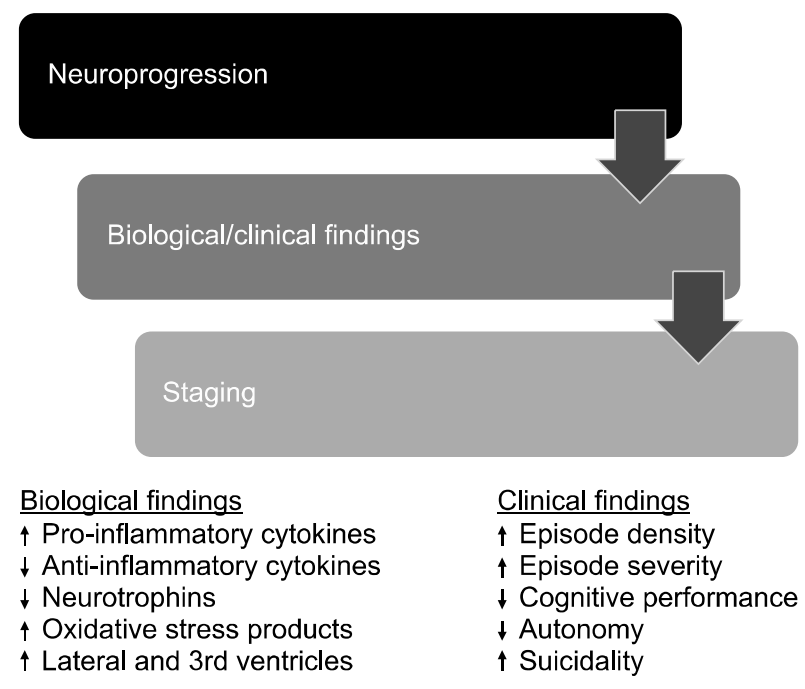

Fig. 3. Linking neurobiological and clinical findings to neuroprogression and staging. 


\section{CNS function}

Non-remitting mood episodes

Addiction

Treatment resistance
Metabolism

Diabetes mellitus

Obesity

Dyslipidemia

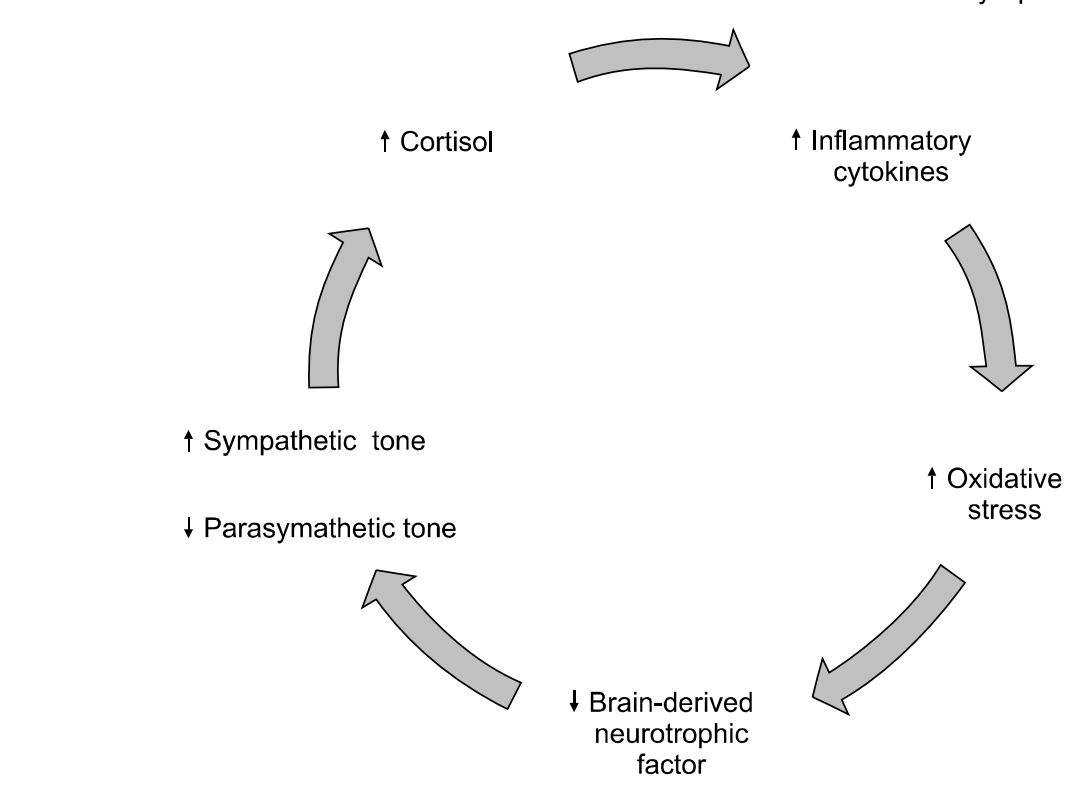

Cardiovascular function

Endothelial cell damage

Atherosclerosis
Immune function

Immune suppression

Auto-immune diseases
Fig. 4. The mediators of allostatic oad act in a vicious cycle in bipolar disorder. CNS, central nervous system. the brain structures of patients with BD. Morphometric studies found enlargements of lateral and 3rd ventricles which were not evident from the onset of the disease and were often found only after several mood episodes. Strakowski et al $^{45)}$ reported that the volume of the ventricles in $\mathrm{BD}$ patients during the first manic episode was similar to that of healthy individuals but lateral ventricles were $122 \%$ larger 8 years after disease onset in cases with history of multiple affective episodes. Other studies have found a progressive diminution in hippocampal volume in multiple episode patients. As hippocampal volume was normal at disease outset and decreased only after repeated episodes, this may be a state marker of BD. ${ }^{46)}$ A number of studies have also found a reduction in the volume of some areas of the prefrontal cortex. López-Larson et al. ${ }^{47)}$ found that although the volume of the prefrontal lobe of patients with BD was similar to that of healthy individuals, there were differences in volume of gray matter in both the right and left prefrontal cortex. Their findings also showed that disease duration might be correlated with a smaller volume of gray matter in the left inferior prefrontal cortex. Finally, many studies have documented the existence of white matter hyperintensities in individuals with $\mathrm{BD}$. The presence of these abnormalities in deep white matter is about 2.5 times more likely to be found in BD patients than in healthy controls. ${ }^{48)}$ Gulseren et al. ${ }^{49)}$ reported that the number of total brain hyperintensities increased with the number of manic episodes. The same authors suggested that these lesions should be used as trait markers because white matter hyperintensities were frequent findings in family members who did not have BD.

\section{Neurotrophic factors}

Brain-derived neurotrophic factor (BDNF) has been extensively studied in BD. Neurotrophic factors are essential for neuronal differentiation and survival and play a vital role in dendritic growth, synptogenesis, and processes like long-term potentiation. BDNF is highly concentrated in limbic and paralimbic areas and its role in the pathogenesis of BD has been well investigated. It is generally believed that serum BDNF levels correlate with brain BDNF concentrations, so that peripheral fluctuations are a sensitive marker for disease activity. Two recent metaanalyses indicated that patients in the manic or depressive phase had lower blood concentrations of BDNF than healthy individuals, and that serum BDNF levels in euthymic patients were not significantly different from those found in controls. ${ }^{50,51)}$ Reduction in serum BDNF level may be a potential biomarker of acute episodes and could differentiate patients in a manic or depressive state from 
those in a euthymic phase at a sensitivity of $90 \%$ and a specificity of $85 \%{ }^{50)}$

In contrast, a recent study found that laboratory measurements of serum BDNF concentrations may distinguish bipolar from unipolar depression at a diagnostic accuracy of $88 \%$ for values equal to or lower than $0.26 \mathrm{pg} / \mathrm{ml}$. The authors compared plasma BDNF concentrations of patients with BD in a depressive state with patients with unipolar depression, and found that the first group expressed lower levels of BDNF. ${ }^{52)}$ Clinically, the difference between bipolar and unipolar depression remains challenging, as the BD diagnosis can only be made if the patient has at least one manic or hypomanic episode. Therefore, blood BDNF concentrations may be an important element to establish the differential diagnosis between these conditions. Other studies suggest that BDNF may be a biomarker of disease progression and severity. In this sense, BDNF values are inversely correlated with severity of symptoms of patients with BD. ${ }^{53)}$ To explore the potential of blood BDNF to discriminate advanced and initial BD states, researchers showed that serum BDNF concentrations may discriminate patients with BD for less than 3 years from patients that have had the disease for over 10 years, at a sensitivity of $100 \%$, specificity of $88 \%$ and accuracy of $95 \%$ for values equal to or lower than 0.62 $\mathrm{pg} / \mathrm{ml}^{54)}$ Another possible use of serum BDNF concentrations is the prediction of response to treatment and the assessment of pharmacological efficacy. In this regard a study found that there is an increase and normalization of blood BDNF levels after appropriate treatment of acute mania. ${ }^{55)}$

\section{Oxidative stress}

Oxidative stress is the result of imbalance between oxidant and antioxidant enzymes, which usually results in cell damage. Lower antioxidant levels and an increased production of pro-oxidant agents give rise to oxidative stress which leads to changes in macromolecules such as lipids, proteins, carbohydrates and DNA. ${ }^{56)}$ The CNS is particularly vulnerable to oxidative injury, because the brain uses a great amount of oxygen which promotes the formation of oxygen free radicals and reactive oxygen species. Moreover, the CNS has a limited antioxidant capacity, and glutathione is the main antioxidant in the brain. ${ }^{57)}$ The enzymes composing the antioxidant system are most importantly superoxide dismutase (SOD), catalase (CAT) and glutathione peroxidase. The role of oxidative stress in the pathophysiology of BD has been investigated in several studies, which consistently report changes in antioxidant enzymes, lipid peroxidation and levels of nitric oxide (NO), an important marker of oxidative stress. ${ }^{58)}$

The meta-analysis conducted by Andreazza et al. ${ }^{59)}$ concluded that some of the oxidative stress markers are elevated in plasma or blood cells of individuals with BD, particularly NO and thiobarbituric acid reactive substances (TBARS), markers of lipid peroxidation. The analysis of blood TBARS concentrations in patients with $\mathrm{BD}$ revealed that these are elevated during all stages of BD. ${ }^{60)}$ This suggests that lipid damage occurs all along the course of this mood disorder. Therefore, serum levels of TBARS may be used as biomarkers of progression of BD provided further studies confirm that these increase with the duration of the disease. In addition to TBARS, NO concentrations are also elevated in $\mathrm{BD}$, regardless of the mood state. ${ }^{61)}$ Andreazza et al. ${ }^{62)}$ found that there are other oxidative stress markers, such as 3-nitrotyrosine which are elevated in the initial stages of BD.

One of the consequences of oxidative stress is DNA damage, and studies with patients with $\mathrm{BD}$ have shown an increase in the frequency of DNA damage. Andreazza $e t$ $a l{ }^{63)}$ described a positive correlation between manic and depressive symptom severity and enhanced DNA damage. At the same time, DNA damage has also been associated with reductions in telomere length in lymphocytes of individuals with $\mathrm{BD}{ }^{64)}$ According to some researchers telomere shortening may be indicative of cumulative oxidative stress. ${ }^{65)}$ Moreover, a study with patients with BD concluded that the number of shortened telomeres and mean telomere length are associated with the number of depressive episodes along life, but not with type of illness. ${ }^{64)}$ Telomere shortening physiologically occurs after each cell division as part of normal ageing. However, telomere shortening found in individuals with BD seems to be indicative of ageing acceleration of the magnitude of about 10 years. These data may explain the association between BD and early medical comorbidities associated with ageing. ${ }^{66)}$

Another element to support the role of oxidative stress in $\mathrm{BD}$ is the evidence that antioxidant compounds, such as $\mathrm{N}$-acetylcystein (NAC) are beneficial in the treatment of $\mathrm{BD}$ according to several studies. NAC, a glutathione precursor efficiently reduces depressive symptoms in patients with BD and improves their functioning and quality of life. ${ }^{67)}$ Treatment effects of mood stabilizers seem to be associated with their action upon oxidative stress pathways. Patients with $\mathrm{BD}$ treated with lithium have a significant reduction of TBARS concentrations and of the SOD/CAT ratio, whereas healthy individuals exposed to 
lithium do not have the same changes. ${ }^{68)}$ These findings suggest that lithium has an antioxidant effect favorable for the treatment of BD. Lastly, to evaluate systemic toxicity in $\mathrm{BD}$, Kapczinski et al. ${ }^{69)}$ compared the concentrations of several markers in patients with $\mathrm{BD}$, healthy individuals and patients with sepsis. One of their notable findings was the impressive scale of oxidative damage during acute BD episodes, as high as the level seen in patients with sepsis in some cases.

\section{Inflammatory factors}

Inflammatory factors also known as cytokines are proteins or glycoproteins secreted by cells of the immune system in response to noxious stimuli. Cytokines gain access to the CNS via the periphery, while microglia the resident brain macrophages also secrete these mediators. Pro-inflammatory cytokines like tumor necrosis factor alpha (TNF- $\alpha$ ) bind to neuronal death receptors and recruit Fas (first apoptosis signal) to activate caspase 8, a pro-apoptotic enzyme leading to cell death. ${ }^{70)}$ The increased neuronal loss via apoptosis has been documented as decrease in hippocampal volume and reduction in prefrontal grey matter in bipolar patients with repeated episodes. Moreover, inflammatory factors interact with HPA axis, autonomic nervous system, and key neurotransmitters like glutamate, serotonin and dopamine. ${ }^{71)}$ Recent studies in bipolar patients have confirmed the presence of a chronic inflammatory state in this mood disorder. ${ }^{72)}$ In general, affective episodes may be classified as pro-inflammatory states, for example one study revealed that patients with a manic exacerbation had higher interleukin-2 (IL-2), IL-4 and IL-6 concentrations than controls, whereas those suffering from bipolar depression only showed elevated levels of IL-6. During euthymic periods only IL-4 levels were raised, suggesting that mania and to lesser degree depression are associated with a proinflammatory state. ${ }^{73)}$ Another study found that patients in a manic state have significantly higher concentrations of IL- 6 and TNF- $\alpha$ than individuals without BD. After 6 weeks of treatment with mood stabilizing drugs, the same patients had normal IL-6 levels, but TNF- $\alpha$ concentrations remained high. Therefore, the authors of this paper suggested that IL-6 might be a marker of manic states. ${ }^{71)}$

At the same time, preliminary studies have found that inflammatory biomarkers are capable of discriminating bipolar patients in the initial stages of the disease from those in advanced stages. According to Kauer-Sant'Anna et al., ${ }^{74)}$ at advanced BD stages serum IL-10 concentrations declined and plasma TNF- $\alpha$ levels increased significantly. Kapczinski et al. ${ }^{54)}$ found that when TNF- $\alpha$ concentrations were equal to or greater than $20.36 \mathrm{pg} / \mathrm{ml}$, patients with BD at advanced stages could be discriminated from patients in initial stages at an accuracy of $91 \%$ and sensitivity and specificity of $97 \%$ and $85 \%$ respectively, indicating that TNF- $\alpha$ might be a potential biomarker of BD progression. In addition to the changes in inflammatory cytokines alluded to above, moderately elevated levels of C-reactive protein are found during manic episodes. $^{75)}$

Increased concentrations of pro-inflammatory cytokines in BD may indicate an association with high rates of medical comorbidities, particularly cardiovascular disease as these inflammatory changes may be risk factors for the development of obesity, hypertension, diabetes and atherosclerosis. ${ }^{76)}$ Such findings stress the importance of medical evaluation and physical management of these patients, with the provision of appropriate and timely treatments to reduce early mortality related to the bipolar diathesis. Comorbidities are considered as not only the consequences of pharmacotherapy and life style characteristics of BD patients, but also a manifestation of this multi-system disease. ${ }^{72)}$ In fact, medical burden is part of the early stages of BD as over $70 \%$ of young individuals are estimated to need treatment for chronic medical conditions. ${ }^{77)}$ Middle-aged patients, in contrast develop heart disease and hypertension at a younger age than adults without $\mathrm{BD}$, underscoring that medical burden should be included in disease staging. ${ }^{78)}$

\section{Staging Models of Bipolar Disorder Based on Neuroprogression and Allostatic Load}

\section{Staging model proposed by Berk et al. ${ }^{79)}$}

BD initiates with an asymptomatic phase (stage 0), which is directly associated with specific risk factors, for example family history of the illness and substance abuse. Stage 1 is divided into $1 \mathrm{a}$ and $1 \mathrm{~b}$, characterized by the presence of mild, nonspecific mood symptoms, with the possibility of evolving to prodromal patterns. Stage 2 is where the 1st episode takes place, in either the manic or depressive phase - the latter being more common. Stage 3, divided into three phases, is characterized by the first relapse or the persistence of subclinical symptoms (phase $3 a)$, by an illness course with borderline symptoms (3b), and by a remission and recurrence pattern (3c). Finally, stage 4 refers to patients who do not achieve full remission of symptoms or are refractory to treatment. 
Staging model proposed by Kapczinski et al, ${ }^{80)}$

This model, proposed some years latter shifts the emphasis to functioning in the inter-episode as a means to assess staging along with the characterization of biomarkers for each phase. The patients are classified into 5 stages, starting with a latent phase in which individuals may experience mood and anxiety symptoms without overt presence of BD. In stage 1, patients already present a period of mania/hypomania but present adequate functioning and absence of cognitive deficits in the inter-episode. In stage 2 , subclinical symptoms, comorbidities and mild cognitive dysfunction are present in the inter-episode. In stage 3 , patients present with marked cognitive and functional impairment. Finally, stage 4 includes individuals with severe cognitive and functional impairment, leading to loss of autonomy. Table 1 gives a comparison of the staging systems for BD along with the putative biomarkers for each stage.

\section{Therapeutic implications of staging}

In other fields of medicine staging is widely used to choose appropriate treatment and inform the prognosis. However, this is a new trend in psychiatry and only recently have the first models of staging of psychiatric disorders been developed. For staging designs to have heuristic value, the course of the disease should be predictable along time and the illness at the initial stages should be associated with simpler treatment requirements and a better outcome. In addition, treatment efficacy should also be greater in the beginning of the ailment and a subsequent progressive decline should be expected, culminating in treatment resistance. For this reason staging formats need to be constructed in parallel to treatment algorithms, as different stages have diverse therapeutic requirements. ${ }^{81)}$ Therefore, it is surmised that staging is instrumental in choosing personalized optimal management plans and is simultaneously helpful in making clear the prospects in individual cases. It must be recognized that early interventions are fundamental to neuroprotection; in addition, while treatment during the initial stages should primarily focus on neuroprotective strategies, therapy during more advanced phases should be palliative and rehabilitative to control the consequences of disease progression. The importance assigned to neuroprotective approaches may take psychiatry to a new era of preventive medicine. ${ }^{82)}$

\section{Limitations of the Staging Models}

The concept of $\mathrm{BD}$ has broadened with the publication of consecutive editions of Diagnostic and Statistical
Manual (DSM). DSM-IV recognized BD type I (manic/ mixed and recurrent MDE), BD type II (hypomanic and recurrent MDE), cyclothymic disorder (hypomanic and recurrent depressive episodes that fall short of MDE) and BD not otherwise specified. However, important changes have been made in DSM-5 with regards to the definition of mixed episodes, as now patients can be diagnosed with suffering from a mixed episode even if they have only a few manic symptoms concurrently with depressive symptoms. Additionally, patients with hypomania can also be identified as having mixed symptoms and a new specifier "with mixed features" has been added to the diagnosis of major depressive disorder (MDD). ${ }^{83)}$ These changes are suggestive of the fact that mood disorders occur on a continuum and the distinction between MDD and BD is blurring. ${ }^{84)}$ The extant literature on the neurobiology of mood disorders indicate that these conditions have common underpinnings and share similar pathophysiologic mechanisms.

The strongest support for the contributory role of inflammation in the causation of MDD comes from the observation that cytokine immunotherapy based on chronic administration of interferon-alpha and/or IL-2 to patients with kidney cancer or melanoma with metastasis induces an episode of depression in a significant proportion of patients. ${ }^{85)}$ Further, anti-TNF $\alpha$ treatment with etanercept and infliximab is emerging as a possible option in the management of resistant mood disorders, either as mono or adjunctive therapy. ${ }^{86)}$ Other lines of evidence show that akin to BD, MDD is a neuroprogressive condition with declining neurotrophic support, augmented neuronal loss in key mood regulating areas like the hippocampus and incremental damage to central and peripheral organs with illness advancement. ${ }^{87)}$ As such attempts have been made to stage MDD; further details on this subject can be obtained from the excellent review by Ferensztajn et al. ${ }^{88)}$ In summary, it can be enunciated that while there are phenotypic differences in major mood disorders, convergent pathophysiological mechanisms operate that lead to the expression of these conditions in predisposed individuals.

As far as the staging models in $\mathrm{BD}$ are concerned the following points need to be highlighted.

i) A developmental clinical staging approach represents an important advance supporting earlier and more accurate psychiatric diagnosis.

ii) Clinical staging of BD must consider both the natural history and the heterogeneity of the diagnosis.

iii) A comprehensive evidence-based staging model of $\mathrm{BD}$ is important to promote early detection and inter- 


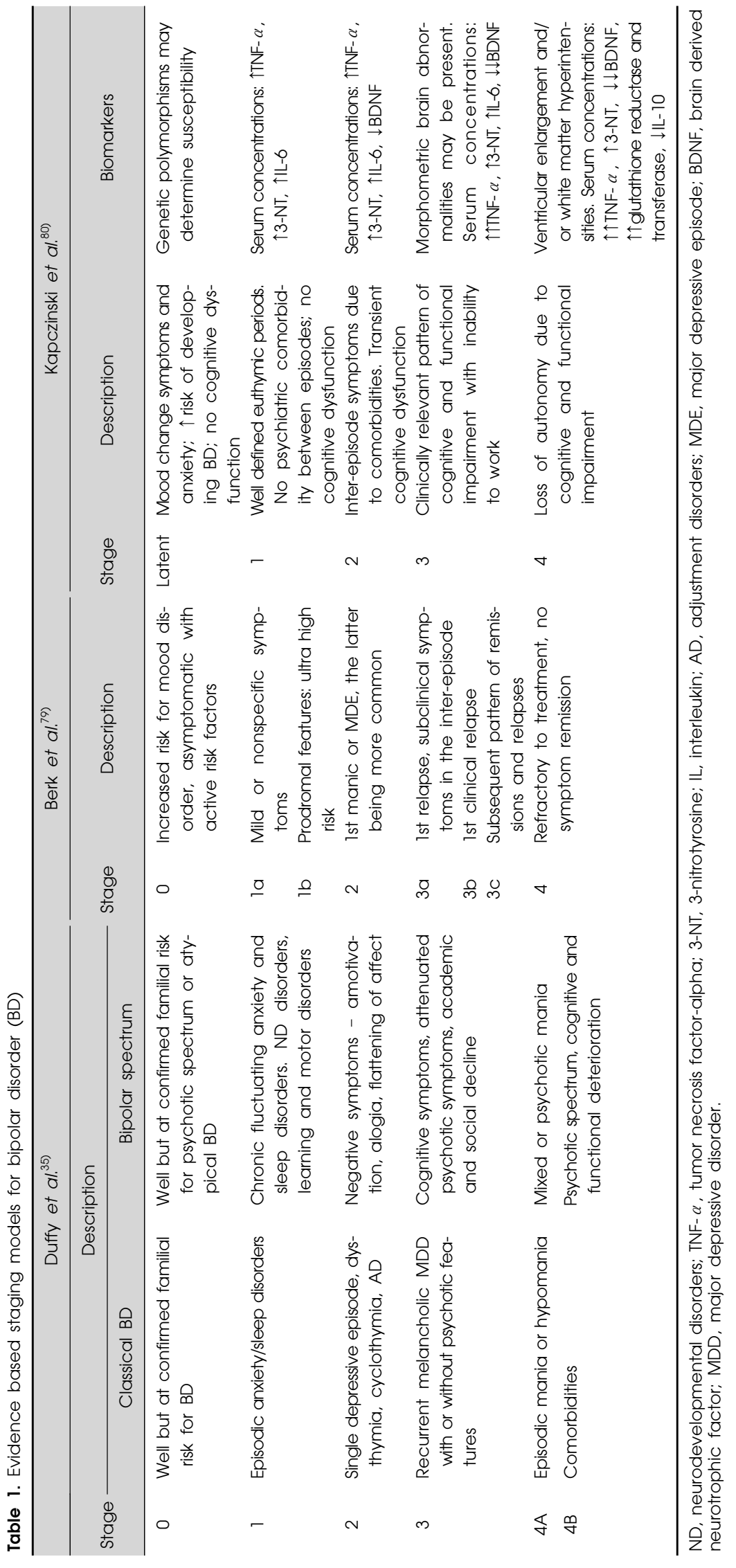


vention, as well as research aimed at identifying markers of illness predisposition and progression.

iv) The proponents of staging BD on the basis of neuroprogression and AL have focused on differentiating earlier- from later-stage illness in adult patients meeting full diagnostic criteria for the disorder.

v) These studies consider neither the evidence of early antecedent risk syndromes nor the developmental illness trajectory leading up to the full-blown diagnosis of BD, as reported in several large prospective studies of the offspring of parents with this condition. ${ }^{27)}$

vi) The proposed staging models should be examined with the caveat that these provide an aggregate view derived from research studies and will not apply to all high-risk offspring as environmental and epigenetic factors operate in the expression of the diathesis.

vii) The problem with AL model proposed by Kapczinski et al. ${ }^{80)}$ is that it focuses solely on progression of end-stage established $\mathrm{BD}$, rather than articulating a staging model based on the full natural history of illness evolution.

viii) While Berk et al.'s ${ }^{79)}$ approach is more comprehensive, it collapses numerous discrete early clinical stages of illness development into a single very heterogeneous stage 1 . Further, it does not incorporate family history as a way to identifying stage 0 high-risk individuals, although a positive family history is the most robust risk factor predicting for at least classical BD. Without the context of positive family history, the early stages are too nonspecific and would yield a very high rate of false positives.

ix) Finally, the staging models will need to be refined and informed by future research advances to be of heuristic value.

\section{CONCLUSION}

Staging of major psychiatric disorders is gaining momentum as the clinical significance of this framework is substantial. Several lines of research show that the course of principal mental disorders follows a predictable and evolving path, from milder to progressively more severe presentations; staging models foster better understanding of the trajectory of these illnesses. BD is a persistent, lifelong condition whose pathogenesis involves the interplay between dysfunctional genes and environmental factors; this interaction is expressed clinically as distinctly recognizable endophenotypes. While the blueprint of any staging system does not of necessity imply that patients would pass through each phase sequentially, it does give an ag- gregate view of how the illness unfolds as it develops. The extrapolative nature of the staging schema helps in formulating individually tailored treatment plans and defining prognosis in patients with BD. Appropriate and adequate treatment in the early phases should prevent advancement to the latter periods of the disease when lasting damage has in essence occurred. In susceptible individuals with an ultra high risk profile, interventions in the earliest stages give an opportunity to prevent the ailment from progressing and resulting in a whole host of undesirable sequelae. Each one of the existing staging models for BD has its merits and limitations, but unquestionably there is a great need for this proposal. It is expected that future research will lead to refinements in the staging protocols with better outcomes for the sufferers of BD, which indeed is an intractable condition with poor prospects for many patients.

\section{REFERENCES}

1. Gonzalez R. The relationship between bipolar disorder and biological rhythms. J Clin Psychiatry 2014;75:e323-e331.

2. Nivoli AM, Murru A, Pacchiarotti I, Valenti M, Rosa AR, Hidalgo D, et al. Bipolar disorder in the elderly: a cohort study comparing older and younger patients. Acta Psychiatr Scand 2014;130:364-373.

3. Fagiolini A, Forgione R, Maccari M, Cuomo A, Morana B, Dell'Osso MC, et al. Prevalence, chronicity, burden and borders of bipolar disorder. J Affect Disord 2013;148:161169.

4. Knežević V, Nedić A. Influence of misdiagnosis on the course of bipolar disorder. Eur Rev Med Pharmacol Sci 2013;17:1542-1545.

5. Leboyer M, Kupfer DJ. Bipolar disorder: new perspectives in health care and prevention. J Clin Psychiatry 2010;71: 1689-1695.

6. Barbosa IG, Bauer ME, Machado-Vieira R, Teixeira AL. Cytokines in bipolar disorder: paving the way for neuroprogression. Neural Plast 2014;2014:360481.

7. Conus P, Macneil C, McGorry PD. Public health significance of bipolar disorder: implications for early intervention and prevention. Bipolar Disord 2014;16:548-556.

8. Frank E, Nimgaonkar VL, Phillips ML, Kupfer DJ. All the world's a (clinical) stage: rethinking bipolar disorder from a longitudinal perspective. Mol Psychiatry 2015;20:23-31.

9. Kapczinski F, Magalhães PV, Balanzá-Martinez V, Dias VV, Frangou S, Gama CS, et al. Staging systems in bipolar disorder: an International Society for Bipolar Disorders Task Force Report. Acta Psychiatr Scand 2014;130:354363.

10. Duffy A, Carlson GA. How does a developmental perspective inform us about the early Natural History of Bipolar Disorder? J Can Acad Child Adolesc Psychiatry 2013;22:6-12.

11. Zivanovic O, Nedic A. Kraepelin's concept of manic-depressive insanity: one hundred years later. J Affect Disord 2012;137: 15-24.

12. McGorry P, Keshavan M, Goldstone S, Amminger P, Allott $\mathrm{K}$, Berk M, et al. Biomarkers and clinical staging in psychiatry. World Psychiatry 2014;13:211-223.

13. Berk M, Hallam K, Malhi GS, Henry L, Hasty M, Macneil 
$\mathrm{C}$, et al. Evidence and implications for early intervention in bipolar disorder. J Ment Health 2010;19:113-126.

14. Rybakowski JK. Response to lithium in bipolar disorder: clinical and genetic findings. ACS Chem Neurosci 2014;5: 413-421.

15. Rybakowski JK. Factors associated with lithium efficacy in bipolar disorder. Harv Rev Psychiatry 2014;22:353-357.

16. Duffy A. The early natural history of bipolar disorder: what we have learned from longitudinal high-risk research. Can $J$ Psychiatry 2010;55:477-485.

17. Grof P. Sixty years of lithium responders. Neuropsychobiology 2010;62:8-16

18. Grof P, Duffy A, Alda M, Hajek T. Lithium response across generations. Acta Psychiatr Scand 2009;120:378-385.

19. Garnham J, Munro A, Slaney C, Macdougall M, Passmore $\mathrm{M}$, Duffy A, et al. Prophylactic treatment response in bipolar disorder: results of a naturalistic observation study. J Affect Disord 2007;104:185-190.

20. Duffy A, Alda M, Kutcher S, Cavazzoni P, Robertson C, Grof $\mathrm{E}$, et al. A prospective study of the offspring of bipolar parents responsive and nonresponsive to lithium treatment. J Clin Psychiatry 2002;63:1171-1178.

21. Alda M. The phenotypic spectra of bipolar disorder. Eur Neuropsychopharmacol 2004;14 Suppl 2:S94-S99.

22. Duffy A, Horrocks J, Doucette S, Keown-Stoneman C, McCloskey S, Grof P. The developmental trajectory of bipolar disorder. Br J Psychiatry 2014;204:122-128.

23. Duffy A, Alda M, Hajek T, Grof P. Early course of bipolar disorder in high-risk offspring: prospective study. Br J Psychiatry 2009;195:457-458.

24. Goldstein BI, Shamseddeen W, Axelson DA, Kalas C, Monk $\mathrm{K}$, Brent DA, et al. Clinical, demographic, and familial correlates of bipolar spectrum disorders among offspring of parents with bipolar disorder. J Am Acad Child Adolesc Psychiatry 2010;49:388-396.

25. Levy B, Manove E. Functional outcome in bipolar disorder: the big picture. Depress Res Treat 2012;2012:949248.

26. Berk M, Kapczinski F, Andreazza AC, Dean OM, Giorlando $\mathrm{F}$, Maes $\mathrm{M}$, et al. Pathways underlying neuroprogression in bipolar disorder: focus on inflammation, oxidative stress and neurotrophic factors. Neurosci Biobehav Rev 2011;35: 804-817.

27. Mesman E, Nolen WA, Reichart CG, Wals M, Hillegers MH. The Dutch bipolar offspring study: 12-year follow-up. Am J Psychiatry 2013;170:542-549.

28. Sparks GM, Axelson DA, Yu H, Ha W, Ballester J, Diler RS, et al. Disruptive mood dysregulation disorder and chronic irritability in youth at familial risk for bipolar disorder. $J$ Am Acad Child Adolesc Psychiatry 2014;53:408-416.

29. Masi G, Mucci M, Pfanner C, Berloffa S, Magazù A, Perugi G. Developmental pathways for different subtypes of earlyonset bipolarity in youths. J Clin Psychiatry 2012;73:13351341.

30. Singh MK, Ketter T, Chang KD. Distinguishing bipolar disorder from other psychiatric disorders in children. Curr Psychiatry Rep 2014;16:516.

31. McNamara RK, Nandagopal JJ, Strakowski SM, DelBello MP. Preventative strategies for early-onset bipolar disorder: towards a clinical staging model. CNS Drugs 2010;24:983996.

32. Duffy A, Alda M, Crawford L, Milin R, Grof P. The early manifestations of bipolar disorder: a longitudinal prospective study of the offspring of bipolar parents. Bipolar Disord 2007:9:828-838.

33. Duffy A, Alda M, Milin R, Grof P. A consecutive series of treated affected offspring of parents with bipolar disorder: is response associated with the clinical profile? Can J Psychiatry 2007;52:369-376.

34. Faedda GL, Marangoni C, Serra G, Salvatore P, Sani G, Vázquez GH, et al. Precursors of bipolar disorders: a systematic literature review of prospective studies. $J$ Clin Psychiatry 2015;76:614-624.

35. Duffy A. Toward a comprehensive clinical staging model for bipolar disorder: integrating the evidence. Can J Psychiatry 2014:59:659-666.

36. Valentí M, Pacchiarotti I, Undurraga J, Bonnín CM, Popovic $\mathrm{D}$, Goikolea JM, et al. Risk factors for rapid cycling in bipolar disorder. Bipolar Disord 2015;17:549-559.

37. Tidemalm D, Haglund A, Karanti A, Landén M, Runeson B. Attempted suicide in bipolar disorder: risk factors in a cohort of 6086 patients. PLoS One 2014;9:e94097.

38. Kim MD, Seo HJ, Yun HJ, Jung YE, Park JH, Lee CI, et al. The relationship between cognitive decline and psychopathology in patients with schizophrenia and bipolar disorder. Clin Psychopharmacol Neurosci 2015;13:103-108.

39. Gama CS, Kunz M, Magalhães PV, Kapczinski F. Staging and neuroprogression in bipolar disorder: a systematic review of the literature. Rev Bras Psiquiatr 2013;35:70-74.

40. Fries GR, Pfaffenseller B, Stertz L, Paz AV, Dargél AA, Kunz $\mathrm{M}$, et al. Staging and neuroprogression in bipolar disorder. Curr Psychiatry Rep 2012;14:667-675.

41. Bizik G, Picard M, Nijjar R, Tourjman V, McEwen BS, Lupien SJ, et al. Allostatic load as a tool for monitoring physiological dysregulations and comorbidities in patients with severe mental illnesses. Harv Rev Psychiatry 2013;21: 296-313.

42. Grande I, Magalhães PV, Kunz M, Vieta E, Kapczinski F. Mediators of allostasis and systemic toxicity in bipolar disorder. Physiol Behav 2012;106:46-50.

43. Kapczinski F, Vieta E, Andreazza AC, Frey BN, Gomes FA, Tramontina $\mathrm{J}$, et al. Allostatic load in bipolar disorder: implications for pathophysiology and treatment. Neurosci Biobehav Rev 2008;32:675-692.

44. McEwen BS. Protective and damaging effects of stress mediators: central role of the brain. Dialogues Clin Neurosci 2006;8:367-381.

45. Strakowski SM, DelBello MP, Zimmerman ME, Getz GE, Mills NP, Ret J, et al. Ventricular and periventricular structural volumes in first-versus multiple-episode bipolar disorder. Am J Psychiatry 2002;159:1841-1847.

46. Moorhead TW, McKirdy J, Sussmann JE, Hall J, Lawrie SM, Johnstone EC, et al. Progressive gray matter loss in patients with bipolar disorder. Biol Psychiatry 2007;62:894900.

47. López-Larson MP, DelBello MP, Zimmerman ME, Schwiers ML, Strakowski SM. Regional prefrontal gray and white matter abnormalities in bipolar disorder. Biol Psychiatry 2002;52:93-100.

48. Kempton MJ, Geddes JR, Ettinger U, Williams SC, Grasby PM. Meta-analysis, database, and meta-regression of 98 structural imaging studies in bipolar disorder. Arch Gen Psychiatry 2008;65:1017-1032.

49. Gulseren S, Gurcan M, Gulseren L, Gelal F, Erol A. T2 hyperintensities in bipolar patients and their healthy siblings. Arch Med Res 2006;37:79-85.

50. Fernandes BS, Gama CS, Ceresér KM, Yatham LN, Fries GR, Colpo G, et al. Brain-derived neurotrophic factor as a state-marker of mood episodes in bipolar disorders: a systematic review and meta-regression analysis. $J$ Psychiatr Res 2011;45:995-1004. 
51. Lin PY. State-dependent decrease in levels of brain-derived neurotrophic factor in bipolar disorder: a meta-analytic study. Neurosci Lett 2009;466:139-143.

52. Fernandes BS, Gama CS, Kauer-Sant'Anna M, Lobato MI, Belmonte-de-Abreu P, Kapczinski F. Serum brain-derived neurotrophic factor in bipolar and unipolar depression: a potential adjunctive tool for differential diagnosis. J Psychiatr Res 2009;43:1200-1204.

53. Grande I, Fries GR, Kunz M, Kapczinski F. The role of $B D N F$ as a mediator of neuroplasticity in bipolar disorder. Psychiatry Investig 2010;7:243-250.

54. Kapczinski F, Fernandes BS, Kauer-Sant'Anna M, Gama CS, Yatham LN, Berk M. The concept of staging in bipolar disorder: the role of BDNF and TNF-alpha as biomarkers. Acta Neuropsychiatr 2009;21:272-274.

55. Tramontina JF, Andreazza AC, Kauer-Sant'anna M, Stertz L, Goi J, Chiarani F, et al. Brain-derived neurotrophic factor serum levels before and after treatment for acute mania. Neurosci Lett 2009;452:111-113.

56. Pfaffenseller B, Fries GR, Wollenhaupt-Aguiar B, Colpo GD, Stertz L, Panizzutti B, et al. Neurotrophins, inflammation and oxidative stress as illness activity biomarkers in bipolar disorder. Expert Rev Neurother 2013;13:827-842.

57. Chitty KM, Lagopoulos J, Hickie IB, Hermens DF. A longitudinal proton magnetic resonance spectroscopy study investigating oxidative stress as a result of alcohol and tobacco use in youth with bipolar disorder. J Affect Disord 2015;175:481-487.

58. Teixeira AL, Barbosa IG, Machado-Vieira R, Rizzo LB, Wieck A, Bauer ME. Novel biomarkers for bipolar disorder. Expert Opin Med Diagn 2013;7:147-159.

59. Andreazza AC, Kauer-Sant'anna M, Frey BN, Bond DJ, Kapczinski F, Young LT, et al. Oxidative stress markers in bipolar disorder: a meta-analysis. J Affect Disord 2008;111: 135-144.

60. Andreazza AC, Cassini C, Rosa AR, Leite MC, de Almeida LM, Nardin P, et al. Serum S100B and antioxidant enzymes in bipolar patients. J Psychiatr Res 2007;41:523-529.

61. Savas HA, Gergerlioglu HS, Armutcu F, Herken H, Yilmaz $\mathrm{HR}$, Kocoglu E, et al. Elevated serum nitric oxide and superoxide dismutase in euthymic bipolar patients: impact of past episodes. World J Biol Psychiatry 2006;7:51-55.

62. Andreazza AC, Kapczinski F, Kauer-Sant'Anna M, Walz JC, Bond DJ, Gonçalves CA, et al. 3-Nitrotyrosine and glutathione antioxidant system in patients in the early and late stages of bipolar disorder. J Psychiatry Neurosci 2009;34: 263-271.

63. Andreazza AC, Frey BN, Erdtmann B, Salvador M, Rombaldi F, Santin A, et al. DNA damage in bipolar disorder. Psychiatry Res 2007;153:27-32.

64. Elvsåshagen T, Vera E, Bøen E, Bratlie J, Andreassen OA, Josefsen D, et al. The load of short telomeres is increased and associated with lifetime number of depressive episodes in bipolar II disorder. J Affect Disord 2011;135:43-50.

65. Saretzki G, Von Zglinicki T. Replicative aging, telomeres, and oxidative stress. Ann N Y Acad Sci 2002;959:24-29.

66. Simon NM, Smoller JW, McNamara KL, Maser RS, Zalta $\mathrm{AK}$, Pollack $\mathrm{MH}$, et al. Telomere shortening and mood disorders: preliminary support for a chronic stress model of accelerated aging. Biol Psychiatry 2006;60:432-435.

67. Magalhães PV, Dean OM, Bush AI, Copolov DL, Malhi GS, Kohlmann $\mathrm{K}$, et al. $\mathrm{N}$-acetylcysteine for major depressive episodes in bipolar disorder. Rev Bras Psiquiatr 2011;33: 374-378.

68. Khairova R, Pawar R, Salvadore G, Juruena MF, de Sousa
RT, Soeiro-de-Souza MG, et al. Effects of lithium on oxidative stress parameters in healthy subjects. Mol Med Rep 2012;5:680-682.

69. Kapczinski F, Dal-Pizzol F, Teixeira AL, Magalhaes PV, Kauer-Sant'Anna M, Klamt F, et al. A systemic toxicity index developed to assess peripheral changes in mood episodes. Mol Psychiatry 2010;15:784-786.

70. Brietzke E, Kapczinski F. TNF-alpha as a molecular target in bipolar disorder. Prog Neuropsychopharmacol Biol Psychiatry 2008;32:1355-1361.

71. Kim YK, Jung HG, Myint AM, Kim H, Park SH. Imbalance between pro-inflammatory and anti-inflammatory cytokines in bipolar disorder. J Affect Disord 2007;104:91-95.

72. Leboyer M, Soreca I, Scott J, Frye M, Henry C, Tamouza $\mathrm{R}$, et al. Can bipolar disorder be viewed as a multi-system inflammatory disease? J Affect Disord 2012;141:1-10.

73. Hamdani N, Doukhan R, Kurtlucan O, Tamouza R, Leboyer M. Immunity, inflammation, and bipolar disorder: diagnostic and therapeutic implications. Curr Psychiatry Rep 2013;15: 387.

74. Kauer-Sant'Anna M, Kapczinski F, Andreazza AC, Bond DJ, Lam RW, Young LT, et al. Brain-derived neurotrophic factor and inflammatory markers in patients with early- vs. latestage bipolar disorder. Int J Neuropsychopharmacol 2009; 12:447-458.

75. Dickerson F, Stallings C, Origoni A, Boronow J, Yolken R. Elevated serum levels of C-reactive protein are associated with mania symptoms in outpatients with bipolar disorder. Prog Neuropsychopharmacol Biol Psychiatry 2007;31:952955.

76. Drake C, Boutin H, Jones MS, Denes A, McColl BW, Selvarajah JR, et al. Brain inflammation is induced by comorbidities and risk factors for stroke. Brain Behav Immun 2011;25:1113-1122.

77. Evans-Lacko SE, Zeber JE, Gonzalez JM, Olvera RL. Medical comorbidity among youth diagnosed with bipolar disorder in the United States. J Clin Psychiatry 2009;70: 1461-1466.

78. Goldstein BI, Kemp DE, Soczynska JK, McIntyre RS. Inflammation and the phenomenology, pathophysiology, comorbidity, and treatment of bipolar disorder: a systematic review of the literature. J Clin Psychiatry 2009;70:10781090.

79. Berk M, Hallam KT, McGorry PD. The potential utility of a staging model as a course specifier: a bipolar disorder perspective. J Affect Disord 2007;100:279-281.

80. Kapczinski F, Dias VV, Kauer-Sant'Anna M, Frey BN, Grassi-Oliveira R, Colom F, et al. Clinical implications of a staging model for bipolar disorders. Expert Rev Neurother 2009:9:957-966.

81. Berk M, Berk L, Dodd S, Cotton S, Macneil C, Daglas R, et al. Stage managing bipolar disorder. Bipolar Disord 2014; 16:471-477.

82. Berk M, Malhi GS, Hallam K, Gama CS, Dodd S, Andreazza AC, et al. Early intervention in bipolar disorders: clinical, biochemical and neuroimaging imperatives. J Affect Disord 2009;114:1-13.

83. McIntyre RS, Soczynska JK, Cha DS, Woldeyohannes HO, Dale RS, Alsuwaidan MT, et al. The prevalence and illness characteristics of DSM-5-defined "mixed feature specifier" in adults with major depressive disorder and bipolar disorder: Results from the International Mood Disorders Collaborative Project. J Affect Disord 2014;172C:259-264.

84. Benazzi F. The continuum/spectrum concept of mood disorders: is mixed depression the basic link? Eur Arch Psy- 
chiatry Clin Neurosci 2006;256:512-515.

85. Musselman D, Royster EB, Wang M, Long Q, Trimble LM, Mann TK, et al. The impact of escitalopram on IL-2-induced neuroendocrine, immune, and behavioral changes in patients with malignant melanoma: preliminary findings. Neuropsychopharmacology 2013;38:1921-1928

86. Raison CL, Rutherford RE, Woolwine BJ, Shuo C, Schettler $\mathrm{P}$, Drake DF, et al. A randomized controlled trial of the tumor necrosis factor antagonist infliximab for treatment- resistant depression: the role of baseline inflammatory biomarkers. JAMA Psychiatry 2013;70:31-41.

87. Moylan S, Maes M, Wray NR, Berk M. The neuroprogressive nature of major depressive disorder: pathways to disease evolution and resistance, and therapeutic implications. Mol Psychiatry 2013;18:595-606.

88. Ferensztajn E, Remlinger-Molenda A, Rybakowski J. Staging of unipolar affective illness. Psychiatr Pol 2014;48:11271141. 